\title{
ASO Author Reflections: Focusing on the Anastomosis or the Other Way Around?
}

\author{
Chao-Chun Chang, $\mathrm{MD}^{1,2}$, Wen-Ruei Tang, $\mathrm{MD}^{1}$, and Yi-Ting Yen, $\mathrm{MD}, \mathrm{PhD}^{1,2}$ \\ ${ }^{1}$ Division of Thoracic Surgery, Department of Surgery, National Cheng Kung University Hospital, Tainan, Taiwan; \\ ${ }^{2}$ Division of Trauma and Acute Care Surgery, Department of Surgery, National Cheng Kung University Hospital, Tainan, \\ Taiwan
}

\section{PAST}

For patients with synchronous hypopharyngeal and esophageal cancer, total pharyngolaryngectomy with esophagectomy is indicated to eradicate both malignancies at the same time. Nevertheless, anastomotic leakage remains an important issue after gastric tube pull-up for alimentary tract reconstruction after the surgery. ${ }^{1} \mathrm{With}$ the improvement of surgical technology and perioperative care, various methods and equipment have been developed to decrease the risk of anastomotic leakage and enhance tissue healing. ${ }^{2,3}$ The role of negative pressure wound therapy has not been evaluated in these patients on a larger time scale.

\section{PRESENT}

This study was approved by the institutional review board of National Cheng Kung University Hospital (A-ER109-421). In their report, ${ }^{4}$ the authors propose an algorithmic approach using continuous negative pressure wound therapy (NPWT) immediately after the surgery to prevent deadly complications secondary to anastomotic leakage. By facilitating the tissue healing process and

This study was approved by the institutional review board of National Cheng Kung University Hospital (A-ER-109-421).

(C) Society of Surgical Oncology 2021

First Received: 8 June 2021

Accepted: 8 June 2021;

Published Online: 30 June 2021

Y.-T. Yen, MD, PhD

e-mail: b85401067@gmail.com enhancing early mobilization, this approach decreased the chance of leakage-associated morbidity and collectively improved overall survival.

\section{FUTURE}

This study ${ }^{4}$ demonstrated the survival benefit of NPWT. Nonetheless, anastomotic leakage, especially delayed leaks, still matters. Further research is warranted to deal with this issue. The promising results of this study should be confirmed in large prospective series.

DISCLOSURE There are no conflicts of interest.

\section{REFERENCES}

1. Dai Z, He Q, Pan B, Liu L, Zhou D. Postoperative complication assessments of different reconstruction procedures after total pharyngolaryngoesophagectomy: tubular gastric pull-up versus whole gastric pull-up. Am Surg. 2018;84:1927-31.

2. Umezawa H, Matsutani T, Yokoshima K, Nakamizo M, Ogawa R. A novel tube-drainage technique of negative pressure wound therapy for fistulae after reconstructive surgery. Plast Reconstr Surg Glob Open. 2018;6:e1885.

3. Teixeira S, Costa J, Bartosch I, Correia B, Silva A. Management of pharyngocutaneous fistula with negative-pressure wound therapy. J Craniofac Surg. 2017;28:e364-7.

4. Chang CC, Wen-Ruei Tang WR, Huang WL, et al. Algorithmic approach with negative pressure wound therapy improved survival in patients with synchronous hypopharyngeal and esophageal cancer undergoing pharyngolaryngoesophagectomy with gastric tube reconstruction. Ann Surg Oncol. 2021. https://doi.org/10.124 5/s10434-021-10365-8.

Publisher's Note Springer Nature remains neutral with regard to jurisdictional claims in published maps and institutional affiliations. 